BULL. AUSTRAL. MATH. SOC.

VOL. 23 (198I), 46I-469.

\title{
NOT QUITE INNER AUTOMORPHISMS
}

\author{
B.H. NeUMANN
}

\begin{abstract}
A question asked by $G$. Kowol is answered by the construction, to an arbitrarily given natural number $n$, of groups $G$ with automorphisms that agree with inner automorphisms on each set of fewer than $n$ elements of $G$, but fail to agree with any inner automorphism on at least one set of $n$ elements.
\end{abstract}

\section{Introduction}

Burnside asked the question whether an automorphism that maps each element of a group onto a conjugate element must be an inner au.tomorphism, and after "many ineffectual attempts" constructed examples to answer his question in the negative [1]; other such examples were made by Wall [3]. The groups of these examples are finite. If also infinite groups are considered, there are other possibilities of defining outer automorphisms that are in some sense approximately inner, for example "locally inner automorphisms" and "almost inner automorphisms"; see, for example, Liebeck [2]. Gerhard Kowol has asked (oral communication) whether there is a group $G$ with an outer automorphism that coincides on each triplet of elements with an inner automorphism (depending, of course, on the triplet), but that does not agree with any inner automorphism on some quadruplet of elements. This leads naturally to the following definition:

DEFINITION. Let $\phi$ be an automorphism of a group $G$ and let $n$ be

Received 9 January 1981. I thank Dr Gerhard Kowol and Dr Hans Lausch for bringing the question to my attention, and for fruitful discussion. I also record my gratitude to the Department of Mathematics of Monash University for its hospitality while I held a Visiting Lectureship there in the Spring Term (September and October) 1980. 
a cardinal number. Then $\phi$ is not quite inner if to every set $S$ of elements of $G$, of cardinality strictly less than $n$, there is an inner automorphism of $G$ which agrees with $\phi$ on $S$, but there is also a set of elements of $G$, of cardinality $n$, on which $\phi$ does not agree with any inner automorphism.

The cardinal number $n$ should, strictly speaking, be made part of the name (say "n-not quite inner"), but for the sake of simplicity it will be suppressed. If $n=\aleph_{0}$, then $\phi$ is a locally inner automorphism; the case $n=1$ is of no great interest: $\phi$ is then an outer automorphism that does not preserve all classes of conjugate elements. The examples of Burnside [1] and Wall [3] can be shown to exemplify $n=2$ (see the Appendix). For $n=3$ an example has been constructed by G. Kowol (unpublished). I here present examples for all finite $n \geq 2$.

\section{Notational conventions}

Groups are denoted by script capital letters, the carrier (or set of elements) of a group by the same letter in italic capitals. The groups used in the construction are vector spaces, and thus naturally written additively; therefore additive notation will also be used for the nonabelian groups to be constructed; in particular the neutral element is denoted by 0 .

Automorphisms are written as right-hand factors; thus if $\phi$ is an automorphism of the group $G$ and if $g \in G$, then $g \phi$ is the image of $g$ under $\phi$. The same letter is used for an element and for the inner automorphism it induces; thus if $h \in G$, then

$$
g h=-h+g+h \text {. }
$$

The commutator of $g$ and $h$ is written

$$
-g-h+g+h=g(-1+h),
$$

and correspondingly the commutator of $g$ and the automorphism $\phi$ is

$$
-g+g \phi=g(-1+\phi) \text {. }
$$

The cardinal number $n$ is thought of as finite and fixed. A field $F$ is arbitrarily chosen; its elements, other than 0 and 1 , are denoted by lower case Greek letters. If $F$ is finite, the construction produces 
finite groups.

\section{The construction}

The ingredients in the construction are three vector spaces $A, B, C$ over $F$, of dimensions $\ln (n+1), n, n+1$, respectively; these will be subgroups of the group $G$ to be constructed, and they will generate $G$. Bases are chosen in these spaces, and denoted by

$$
\begin{aligned}
& a_{00}, a_{01}, \ldots, a_{0, n-1}, a_{11}, a_{12}, \ldots, a_{1, n-1}, \ldots, a_{n-2, n-1}, a_{n-1, n-1}, \\
& b_{0}, b_{1}, \ldots, b_{n-1}, \\
& c_{0}, c_{1}, \ldots, c_{n-1}, c_{n},
\end{aligned}
$$

respectively. The commutation rules between these spaces are specified as follows: if $a \in A, b \in B, c \in C$ are arbitrary, then

$$
a(-1+c)=b(-1+c)=0 \text {, }
$$

so that $C$ is central in the group under construction. It remains to specify how $A$ and $B$ commute. If

$$
a=\sum_{i=0}^{n-1} \sum_{j=i}^{n-1} \alpha_{i j} a_{i j}, \quad b=\sum_{i=0}^{n-1} \beta_{i} b_{i},
$$

put

$$
a(-1+b)=\sum_{i=0}^{n-1}\left(\sum_{i=j}^{n-1} \alpha_{i j}{ }_{j}\right) c_{i}+\left(\sum_{i=0}^{n-1} \alpha_{i i} \sum_{i=0}^{n-1} \beta_{i}\right) c_{n} .
$$

The rules (3.1) and (3.2), together with the obvious composition rules within each of the three vector spaces, suffice to define the group $G$; they are easily seen to be consistent and to make $G$ nilpotent of class 2. If

$$
\begin{aligned}
& g=\sum_{i=0}^{n-1} \sum_{j=i}^{n-1} \alpha_{i j} a_{i j}+\sum_{i=0}^{n-1} \beta_{i} b_{i}+c, \\
& h=\sum_{i=0}^{n-1} \sum_{j=i}^{n-1} \lambda_{i j} a_{i j}+\sum_{i=0}^{n-1} \mu_{i} b_{i}+d,
\end{aligned}
$$

with $c, d \in C$, are arbitrary elements of $G$, then an easy calculation, not presented here, gives 
(3.5) $g(-1+h)=\sum_{i=0}^{n-1}\left(\sum_{j=i}^{n-1}\left(\alpha_{i j}{ }^{\mu}-\lambda_{i j} \beta_{j}\right)\right) c_{i}$

$$
+\left(\sum_{i=0}^{n-1} \alpha_{i i} \sum_{i=0}^{n-1} \mu_{i}-\sum_{i=0}^{n-1} \lambda_{i i} \sum_{i=0}^{n-1} B_{i}\right) c_{n} .
$$

Define $\phi$ by

$$
g \phi=g+\left(\sum_{i=0}^{n-1} B_{i}\right) c_{n},
$$

where $g$ is given by (3.3). Then $\phi$ is clearly a (central) automorphism of $G$, and

$$
g(-1+\phi)=\left(\sum_{i=0}^{n-1} \beta_{i}\right) c_{n} .
$$

THEOREM. The automorphism $\phi$ of $G$ is not quite inner.

Proof. To show that on every set of fewer then $n$ elements $\phi$ agrees with some inner automorphism, it suffices to deal with sets of $n-1$ elements: smaller sets can be made up to this number. Thus let, for $1 \leq k \leq n-1$,

$$
g^{k}=\sum_{i=0}^{n-1} \sum_{j=i}^{n-1} \alpha_{i j}^{k} a_{i j}+\sum_{i=0}^{n-1} \beta_{i}^{k_{b}}+c^{k},
$$

with $e^{k} \in C$, be $n-1$ arbitrary elements of $G$. Put

$$
b^{k}=\sum_{i=0}^{n-1} \beta_{i}^{k} b_{i}
$$

and choose

$$
b^{0}=\sum_{i=0}^{n-1} \beta_{i}^{0} b_{i} \in B
$$

so that $b^{0}$ is non-zero and orthogonal to $b^{1}, b^{2}, \ldots, b^{n-1}$. Thus

$$
\sum_{i=0}^{n-1} B_{i}^{0} B_{i}^{k}=0 \text { for } 1 \leq k \leq n-1 \text {. }
$$

As $b^{0}$ is non-zero, not all the $\beta_{i}^{0}$ are zero; choose $t$ as the least 
suffix $i$ for which $\beta_{i}^{0} \neq 0$. Next put, for $0 \leq i \leq n-1$,

$$
\beta_{i}^{*}=\frac{1}{\beta_{t}^{0}} \cdot \beta_{i}^{0}
$$

so that $B_{t}^{*}=1$ and

$$
\sum_{i=0}^{n-1} \beta_{i}^{*} \beta_{i}^{k}=0 \text { for } 1 \leq k \leq n-1 \text {; }
$$

in fact, as $\beta_{0}^{*}=\ldots=\beta_{t-1}^{*}=0$,

$$
\sum_{j=t}^{n-1} \beta_{j}^{*} \beta_{j}^{k}=0 \text { for } 1 \leq k \leq n-1 \text {. }
$$

Next put

$$
h^{*}=\sum_{j=t}^{n-1}\left(-\beta_{j}^{*}\right) a_{t j} .
$$

In terms of (3.4) this means that $\lambda_{i j}=0$ except when $i=t$, and then $\lambda_{t j}=-\beta_{j}^{*}$; and all $\mu_{i}=0$ and $d=0$. Hence $\sum_{i=0}^{n-1} \lambda_{i i}=-\beta_{t}^{*}=-1$, and (3.5) reduces to

$$
g\left(-1+h^{*}\right)=\left(\sum_{j=t}^{n-1} \beta_{j}^{*} \beta_{j}\right) c_{t}+\left(\sum_{i=0}^{n-1} \beta_{i}\right) c_{n} .
$$

In particular, for $I \leq k \leq n-1$,

$$
g^{k}\left(-1+h^{*}\right)=\left(\sum_{i=0}^{n-1} B_{i}^{k}\right) c_{n}=g^{k}(-1+\phi),
$$

by (3.7) and (3.6). This shows then that on the set of $n-1$ arbitrary elements $g^{1}, g^{2}, \ldots, g^{n-1}$ the automorphism $\phi$ agrees with the inner automorphism induced by $h^{*}$.

On the other hand, consider the $n$-tuplet $b_{0}, b_{1}, \ldots, b_{n-1}$ of basis elements of $B$ and an arbitrary element $h$ given by (3.4). Then, applying (3.5), 


$$
b_{k}(-1+h)=\sum_{i=0}^{n-1}-\lambda_{i k} c_{i}-\left(\sum_{i=0}^{n-1} \lambda_{i i}\right) c_{n},
$$

whereas by $(3.6)$,

$$
b_{k}(-1+\phi)=c_{n} \text {. }
$$

Thus the inner automorphism induced by $h$ agrees with $\phi$ on $b_{k}$ if, and only if,

$$
\sum_{i=0}^{n-1} \lambda_{i i}=-1
$$

and $\lambda_{i k}=0$ for each relevant $i$, that is for $0 \leq i \leq k$. For $h$ to agree with $\phi$ on all $n$ basis elements $b_{0}, b_{1}, \ldots, b_{n-1}$ it would then be necessary that $\lambda_{i k}=0$ for all $i, k$ (with $0 \leq i \leq k \leq n-1$ ), and in particular $\lambda_{i i}=0$ for $0 \leq i \leq n-1$. This is clearly incompatible with (3.8), and it follows that $\phi$ is not quite inner. //

The finiteness of $n$ is not essential in this construction, and it can be used to make examples of groups with locally inner automorphisms that are not inner; but there is no point in this, as much simpler examples are known.

\section{Appendix: The examples of Burnside and Wall}

I paraphrase the example of Burnside [1] to show its similarity with those in the preceding section. Again $F$ is a field, but now it has to be so chosen that the equation

$$
\xi^{2}=2
$$

has no solution in it; Burnside takes a prime field of finite order $p$ such that 2 is not a quadratic residue modulo $p$. The building blocks are again three vector spaces $A, B, C$ over $F$, but this time they are all of 2 dimensions, with bases

$$
a_{0}, a_{1} ; b_{0}, b_{1} ; c_{0}, c_{1},
$$

respectively. The commutation rules are, for $a \in A, b \in B, c \in C$ : 


$$
a(-1+c)=b(-1+c)=0,
$$

so that again $C$ is central in the group $G$ being constructed; and

$$
\begin{aligned}
& a_{0}\left(-1+b_{0}\right)=c_{0} \quad, \quad a_{0}\left(-1+b_{1}\right)=c_{1}, \\
& a_{1}\left(-1+b_{0}\right)=c_{0}+c_{1}, a_{1}\left(-1+b_{1}\right)=c_{0}-c_{1} .
\end{aligned}
$$

These rules again define $G$, and $G$ is again nilpotent of class 2 .

If

$$
\begin{aligned}
& g=\alpha_{0} a_{0}+\alpha_{1} a_{1}+\beta_{0} b_{0}+\beta_{1} b_{1}+c, \\
& h=\lambda_{0} a_{0}+\lambda_{1} a_{1}+\mu_{0} b_{0}+\mu_{1} b_{1}+d,
\end{aligned}
$$

with $c, d \in C$, are two arbitrary elements, then by a short computation, not presented here,

$$
g(-1+h)=\gamma_{0} c_{0}+\gamma_{1} c_{1}
$$

where

$$
\begin{aligned}
& \gamma_{0}=\alpha_{0} \mu_{0}-\beta_{0} \lambda_{0}+\alpha_{1} \mu_{0}-\beta_{0} \lambda_{1}+\alpha_{1} \mu_{1}-\beta_{1} \lambda_{1}, \\
& \gamma_{1}=\alpha_{0} \mu_{1}-\beta_{1} \lambda_{0}+\alpha_{1} \mu_{0}-\beta_{0} \lambda_{1}-\alpha_{1} \mu_{1}+\beta_{1} \lambda_{1} .
\end{aligned}
$$

It is not difficult to determine, for given $g \notin C$, those $h$ that make $\gamma_{0}=\gamma_{1}=0$, that is to say, commute with $g$. Modulo the centre $C$ they form a subspace of $A+B$ of codimension $I$ or 2 , and one can verify that codimension 1 does not, in fact, occur: this stems from the assumption that 2 is not a square in $F$. Thus every element that differs from $g$ by an element of the centre is a conjugate of $g$, and every central automorphism of $G$, that is to say every automorphism that adds only centre elements to each element, has the property that it leaves the classes of conjugate elements invariant. If the order of $F$ is $p$, then there are $p^{8}$ such automorphisms, but only $p^{4}$ of them can be inner. This argument shows not only that there exist outer automorphisms of $G$ that leave all conjugacy classes of elements invariant: it actually shows that there are many of them, namely at least $p^{8}-p^{4}$. Consider, in particular, the pair $b_{0}, b_{1}$, with an arbitrary element 
h. Then

$$
\begin{aligned}
& b_{0}(-1+h)=\left(-\lambda_{0}-\lambda_{1}\right) c_{0}-\lambda_{1} c_{1}, \\
& b_{1}(-1+h)=-\lambda_{1} c_{0}-\left(\lambda_{0}-\lambda_{1}\right) c_{1} .
\end{aligned}
$$

Thus if the automorphism $\phi$ of $G$ is so chosen that

$$
\begin{aligned}
& b_{0}(-1+\phi)=c_{1}, \\
& b_{1}(-1+\phi)=0,
\end{aligned}
$$

say, then no inner automorphism can match $\phi$ on this pair, and $\phi$ is not quite inner, with $n=2$.

The examples of Wall [3] are different: they need not even be nilpotent, and the nilpotent ones have class greater than 2 . They are the holomorphs of finite cyclic groups of order divisible by 8 . The smallest of them has order 32 . It is not difficult to verify that the class preserving outer automorphisms described by Wall do not preserve the conjugacy classes of all pairs of elements, that is to say, they are not quite inner with $n=2$. Burnside's smallest example of this kind has order 729 , while mine, taking $F$ as the field of order 2 , has order $2^{8}=256$. Thus for not quite inner automorphisms with $n=2$, Wall's groups provide much the smallest examples. It would be interesting to know whether his examples can be generalised to groups with not quite inner automorphism for $n>2$, of order smaller than the smallest of mine, which is $2^{\frac{3}{2} n(n+5)+1}$.

ADDENDUM (19 February 1981). Dr Hans Lausch has kindly informed me that the question which I have ascribed to Dr Kowol was in fact first asked, a few years ago, by Professor Wilfried Nöbauer. I apologise to Dr Kowol and Professor Nöbauer for erroneously attributing this question to the one instead of the other.

\section{References}

[1] W. Burnside, "On the outer isomorphisms of a group", Proc. London Math. Soc. (2) 11 (1913), 40-42. 
[2] Hans Liebeck, "Locally inner and almost inner automorphisms", Arch. Math. (Basel) 15 (1964), 18-27.

[3] G.E. Wall, "Finite groups with class-preserving outer automorphisms", J. London Math. Soc. 22 (1947), 315-320 (1948).

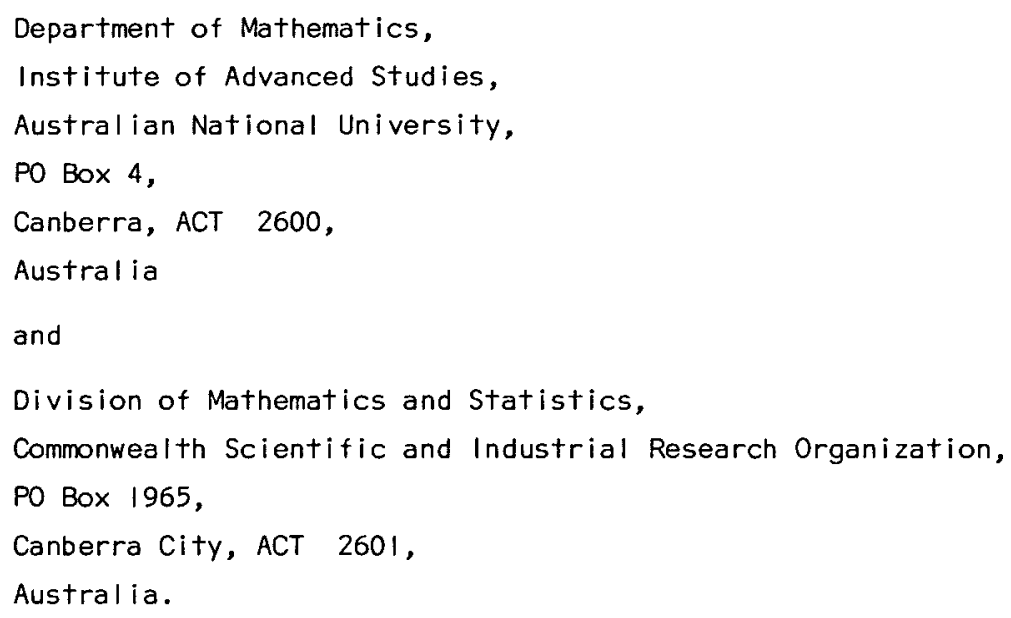

